Digital Press Social Sciences and Humanities

La traduction de l'article défini français en indonésien dans le roman Syngué Sabour par Atiq Rahimi

Laras Intan Taslima and Sajarwa

Proceeding of Conférence internationale sur le français 2018

Joesana Tjahjani, Merry Andriani, Sajarwa, Wening Udasmoro (eds) 


\title{
La traduction de l'article défini français en indonésien dans le roman Syngué Sabour par Atiq Rahimi
}

\author{
Laras Intan Taslima* et Sajarwa \\ Faculty of Cultural Sciences, Universitas Gadjah Mada, Yogyakarta, Indonesia \\ *e-mail : larastas5@gmail.com
}

\section{Résumé}

L'article français appartient au déterminant. C'est très important dans la structure d'une phrase du français. Le français est l'une des langues romanes qui connaît le système d'articles pour chaque nom. Les articles français indiquent le genre et le nombre de nom, donc chaque nom doit être accompagné par un article. L'article défini est le point principal dans cette recherche. L'article défini dans ce roman Syngué Sabour par Atiq Rahimi est traduit en indonésien dans le roman Batu Kesabaran. La traduction du roman Syngué Sabour du français en indonésien est intéressante à parler en raison des différences entre les systèmes déterminants utilisés par les deux langues. La diversité des formes de traduction d'articles français dans le roman de Syngué Sabour sera la principale source de données de cette recherche. Les différentes formes de traduction des articles en langue française seront classées selon la forme de la traduction en indonésien, ayant une équivalence égale ou nulle. Cette recherche vise à déterminer la variété des formes et des facteurs de formation de formes variées de traduction d'articles français dans le roman Syngué Sabour (Pierre de Patience).

\section{Mots-clés}

traduction, article défini, français, Syngué Sabour

\begin{abstract}
French article belongs to the determiner, which has an important role in French sentence structure. French is one of the Romance languages having the article system for each noun. French articles indicate the genre and the number of a noun, so that each noun should be accompanied by an article. The definite article is the main point in this research. The definite article in the novel Syngué Sabour by Atiq Rahimi is translated into Indonesian in the novel Batu Kesabaran. The translation of the novel Syngué Sabour of French into Indonesian is interesting to analyze because of differences of the determiner system both languages. The diversity of translation forms French articles in Syngué Sabour novel is the main source of this research data. The different forms of translation of the articles in French are ranked according to the form of the translation in Indonesian, with an equal equivalent or zero form. This research aims to identify the variety of forms and factors in the formation of various forms of translation of French articles in the novel Syngué Sabour (Pierre de Patience).
\end{abstract}

\section{Keywords}

translation, definite articles, French, Syngué Sabour

\section{Introduction}

La traduction est transférée le message du texte source en autre langue dans la même façon que l'auteur écrit (Newmark, 1988, p. 5). La traduction est intéressante à discuter en raison de la différence entre les deux langues ou plus qui se changer le message. Le point plus important dans traduire le texte est la même compréhension des lecteurs quand ils lisent le texte traduit. Ce but rend des traducteurs de faire leurs meilleurs efforts de transmettre le message entre des langues pour donner le même sens et 
compréhension. En plus, ce point important de traduction montre un problème très cliché dans le domaine de traduction. Ce problème est la variation de choisir les mots pour traduire le texte en raison de tenir le sens et le message dans le texte, particulièrement dans le roman.

Pierre de Patience ou Syngué Sabour est le roman qui est écrit par Atiq Rahimi. Syngué Sabour ça veut dire pierre de patience dans la langue perse. Ce roman raconte sur une femme qui habite avec son mari et ses deux filles dans une ville qui est en guerre. Le point intéressant de ce roman est comment les gens de certaines communautés valorisent une femme comme une commodité qui n'a pas le même droit comme l'homme. Il raconte sur la force de sa société qui la fait une femme construite dans la norme et les valeurs locales. Quand une femme doit obéir son mari n'importe quoi la raison, comment la femme doit obéir ses parents quand elle est demandée de se marier à un homme étrange. Ce roman explique l'inégalité et le suffrage de femme qui n'a pas de voix de donner d'opinion ou vivre sa vie. Cette femme, le caractère principal, elle déteste de rester prendre soin son mari qui est coma. Elle dit ce qu'elle veut tous les jours à son mari parce qu'il est coma et elle n'a personne à parler de ses inquiétudes.

Le problème trouvé dans le roman en termes de traduction est la variation de la traduction de l'article défini dans ce roman. La traduction de l'article défini français en indonésien a la variation basée sur des conditions différentes. Comme dans l'exemple suivant :

(1) Au fond de la chambre, il y a un autre rideau. (Rahimi, 2008, p. 13) Di ujung kamar, tergantung sebuah tirai yang lain. (Rahimi, 2012, p. 1)

Cet exemple est l'exemple quand l'article défini est traduit comme zéro ou il n'y a pas l'autre article en indonésien qui échange l'article défini « la ». C'est le problème qui est discuté dans cet article, comment la traduction entre deux langues qui ont le système différent de nommer leurs noms. La langue indonésienne ne connaît pas le même système du déterminant comme la langue française. Mais la langue indonésienne a des déterminant qui accompagne le nom, mais avec certaine condition. Par exemple dans ce roman, on trouve que la traduction ci-dessous :

(2) La tête de la femme bouge. (Rahimi, 2008, p. 16)

Kepala sang perempuan bergerak. (Rahimi, 2012, p. 4)

On peut voir que la traduction de l'article «la » se traduit comme «sang ». Cet un exemple qui montre l'utilisation du déterminant en indonésien. Même si le déterminant n'est pas toujours ensemble avec le nom, comme dans la langue française. Pour identifier la variation de traduction de l'article défini dans le texte source et le texte cible, tout d'abord on note tous les mots qui ont la justification d'équivalence de traduction. C'est où dans le roman ? Ça se trouve dans chaque phrase qui se traduit dans la langue cible et avoir le même message comme dans le texte source même s'il n’y a pas le mot équivalent ou bien zéro. Avant d'expliquer l'analyse des données, on explique d'abord la théorie sur la traduction, l'équivalence et le déterminant. On va parler beaucoup sur l'article défini et l'utilisation en indonésien regardant les données du roman Syngué Sabour.

\section{Discussion}

\subsection{Le déterminant : L’article défini français en comparaison avec l'article indonésien}

Le déterminant est le particle dans le nom (devant ou derrière du nom) et limiter la définition du nom, par exemple $s i, i t u, n y a, m u$, etc. Le déterminant indéfini dans la langue indonésienne est le déterminant du nom qui donne la définition en général, par exemple suatu (Kridalaksana, 1982, p. 33). Dans la langue française, le déterminant se signifie dans quelques catégories qui se divisent par le nombre du nom et le genre du nom. Le déterminant en français est aussi se catégorie selon la situation plus précis ou plus général. L'un des déterminants qui existe devant le nom est l'article. Cet article qui se divise dans les catégories du nombre et du genre. L'article se situe devant le nom qui signifie le nombre du nom et son genre. En français, l'article est obligatoire devant chaque nom. L'article s'utilise comme un outil pour marquer le nom, ce que c'est défini ou indéfini (Baskoro, 1992, p. 16).

En français, il y a deux types d'article. Ce sont l'article défini et l'article indéfini. Dans cet article on parle de l'article défini pour préciser la catégorie de données et l'analyse. Avant de parler sur l'article défini, c'est mieux de comprendre la différence entre le défini et l'indéfini. Le défini et l'indéfini déterminent la référence du nom. L'utilisation le nom défini ou le nom indéfini se signifie par le locuteur 
et l'interlocuteur, est-ce qu'il y a la même compréhension entre eux et la référence qui réfère au nom. Cette condition ce qu'on appel le contrat de communication (Givon, 1984, p. 399).

L'article défini en français se trouve dans :

a. Le nom de quelqu'un

b. Le nom de quelque chose unique

c. La profession en général

d. Le pronom, le pronom démonstratif et le pronom possessif

e. Le nom partitif

f. Le nom qui est suivi par l'explication

g. Le nom qui est accompagné par le déterminant possessif, l’article défini, le déterminant mélange en avant

h. Zéro

i. Le nom appositive

On peut signifier le nom défini par les identifications ; l'article défini, le déterminant possessif, le déterminant de l'indéfini et du défini, le déterminant du défini et du numéral, le déterminant du numéral cardinal. On peut voir dans la table ci-dessous (Sajarwa, 2003, p. 135)

Tableu 1 L'article en français

\begin{tabular}{|c|c|c|}
\hline $\begin{array}{l}\text { La } \\
\text { catégorie }\end{array}$ & L'article & L'exemple \\
\hline \multirow[t]{3}{*}{ Le défini } & Avec l'article & L'article défini : le, la, les \\
\hline & & $\begin{array}{l}\text { Le déterminant possessif : ma, ta, sa, mon, ton, son, ses, leur, leur, votre, } \\
\text { vos, notre, nos } \\
\text { Le déterminant démonstratif : ce, cet, cette, ces }\end{array}$ \\
\hline & Sans l'article & $\begin{array}{l}\text { Le nom de quelqu'un : Pierre } \\
\text { Le pronom : je, tu, elle, il, nous, vous, les, leur, etc. }\end{array}$ \\
\hline L'indéfini & Avec l'article & L'article indéfini : un, une, des \\
\hline
\end{tabular}

Le défini en indonésien se détermine par l'article défini et l'indéfini se détermine par l'article indéfini dans une forme de classification. L'article défini en indonésien ne montre pas toujours, le nom en indonésien n'utilise pas toujours l'article, ça veut dire que c'est zéro (Baskoro, 1992, p. 518).

\subsection{L'article défini français en comparaison avec l’article en indonésien}

Selon Kaswanti Purwo (1978) dans (Baskoro, 1992), le pronom démonstratif «itu »s'utilise beaucoup pour expliquer le nom qui est explicite. C'est à dire que la référence entre les deux personnes qui se communiquent est claire explicitement. En outre, le pronom possessif «-nya » montre beaucoup dans le nom qui est plus implicite. La différence entre le nom indonésien et le nome français se trouve dans le montant de l'article au nom. La défini et l'indéfini en indonésien ne se montre pas toujours par l'article. Il y a les noms indonésiens qui n'ont pas besoin d'un article parce que l'existence de cet article n'est pas nécessaire.

La caractéristique du nom en indonésien se divise par trois types ci-dessous :

1. Obligatoire

2. Interdit

3. Pas obligatoire

Toutes les catégories au-dessus relient avec l'importance d'un article de chaque nom, n'importe quoi l'information qui est contenu par le nom. On peut dire que la condition du type de l'article en indonésien dépend à la différence entre le locuteur et l'interlocuteur. Le nom qui vient de montrer dans un énoncé n'est pas toujours un nom indéfini. Généralement le nom qui a l'information générique n'a pas besoin d'un article. 
Le nom défini qui montre pour expliquer de mentionner l'information prévue (prior mention) a tendance d'utiliser l'article obligatoirement. C'est pour marquer l'information mentionnée dans le même nom qui a la même référence. Puis, le nom qui n'a pas d'article se montre beaucoup dans le nom générique (le nom qui a l'information comprise par les locuteurs). L'article est injustifié pour ce type du nom. Le nom indéfini en indonésien se montre plus dans l'article zéro ou sans le marqueur (Baskoro, 1992, p. 523). L'article qui n'est pas obligatoire est plus justifié pour le nom générique, parce que son rôle de marquer l'objet (Moeliono, 1988).

La condition définie ou indéfinie d'un nom ne se définit pas par son article. Il se défini par la définition de la référence uniquement dans l'énoncé. Le nom est toujours indéfini s'il n'y a pas l'identification de sa définition. Dans la langue indonésienne, l'article sans marqueur ou zéro n'est pas obligatoire comme dans la langue anglaise et française (Baskoro, 1992).

\subsection{La catégorie de traduction d'article défini en indonésien dans le roman Syngué Sabour}

La méthode qu'on utilise dans cet article est la méthode de lire les déterminants dans le nom qui soit défini ou indéfini. On lit le texte tout d'abord et catégoriser tous les noms selon le type de son déterminant (Sudaryanto, 1993, p. 95). On groupe les noms qui sont traduits en indonésien selon la traduction et la catégorie (définie ou indéfinie) de la langue française. On a 40 phrases comme les données de cet article qui se divise par trois catégories, ce sont la traduction zéro, le pronom et le pronom démonstratif. On utilise les phrases (du texte source) qui montrent la différence de traduction entre l'article de la langue française et la langue indonésienne. La phrase (du texte source) qui est traduite en indonésien (au texte cible) ait l'équivalence ou n'ait pas d'équivalence.

Parmi toutes les 40 phrases, le plus grand nombre s'appartient à la traduction zéro, ou on peut dire il n'y a pas la traduction qui suffit la définition entre les deux langues. Selon les trois catégories, on a la comparaison ci-dessous :

La traduction zéro (n’a pas d'équivalence) $\quad: 28 / 40$

La traduction du pronom (sang, si) $\quad: 11 / 40$

La traduction du pronom démonstratif (itu) $\quad: 9 / 40$

Le pronom démonstratif «itu» s'utilise dans le nom défini (avoir l'explication) et le nombre est singulier. Si le nom est pluriel, on n'utilise pas le pronom «itu». Selon le dictionnaire de l'indonésien (KBBI, 2019), le pronom «itu » est un pronom démonstratif pour définir un objet (le temps, la chose) qui est loin du locuteur. Dans le roman Syngué Sabour, le pronom «itu » montre plus pour identifier une chose qui a été explique en avant ou a été raconte en avant ou en après. On peut voir l'exemple ci-dessous

(3) La chambre est petite. (Rahimi, 2008, p. 13)

Kamar itu kecil. (Rahimi, 2012, p. 1)

Cette phrase est la première phrase dans le paragraphe :

La chambre est petite. Rectangulaire. Elle est étouffante malgré ses murs clairs, couleur cyan, et ses deux rideaux aux motifs d'oiseaux migrateurs figés dans leur élan sur un ciel jaune et bleu. Troués ça et là, ils laissent pénétrer les rayons du soleil pour finir sur les rayures éteintes d'un kilim. Au fond de la chambre. Il y a un autre rideau. Vert. Sans motif aucun. Il cache une porte condamnée. Ou un débarras. (Rahimi, 2008, p. 13)

Le pronom «itu » s'utilise pour être la référence de la chambre qui sera expliqué aux phrases dans le paragraphe. Cet exemple explique que le pronom «itu » est équivalent avec l'article défini «la » dans le nom « chambre » qui est un nom féminin singulier.

Ensuite, l'article défini français dans ce roman montre beaucoup de la traduction du pronom "sang » et «si ». Selon le dictionnaire de l'indonésien (Kemdikbud, 2019), le pronom «sang » est le pronom qui s'utilise pour appeler le nom de quelqu'un, l'animal, ou la chose qui est vivant ou honorable. Le pronom «sang » donne une valeur plus positive pour quelqu'un ou quelque chose et la position plus haute dans la hiérarchie sociale ou dans ce contexte, on peut dire la hiérarchie des caractères. On a l'exemple ci-dessous

(4) La tête de la femme bouge. (Rahimi, 2008, p. 16)

Kepala sang perempuan bergerak. (Rahimi, 2012, p. 4) 
Cette phrase est la première phrase dans le paragraphe :

La tête de la femme bouge. Lasse. Elle quitte le creux de ses genoux.

Il y a un autre exemple pour le pronom "sang" ci-dessous :

(5) La femme retire sa main de la poitrine de l'homme. (Rahimi, 2008, p. 16)

Perempuan itu menarik tangannya dari dada sang lelaki. (Rahimi, 2012, p. 4)

De ces exemples, on peut comparer l'utilisation de pronom "sang" selon son remplacement dans une phrase. Dans l'exemple (4), l'article défini «la » se traduit comme "sang" parce que c'est la première phrase d'un paragraphe. Donc, la fonction est pour donner la définition plus précise à la femme. Pourquoi on ne le traduit au pronom démonstratif «itu »? C'est pour donner la concentration à la femme, que cette femme est importante dans cette partie, cette partie raconte plus de cette femme. Le pronom "sang " donne une valeur de l'importance de ce caractère pour être la. Les phrases après, ce sont les phrases d'explication de la première phrase qui ne raconte que la femme. En autre côté, la traduction de l'article défini «la » au pronom "sang" s'utilise aussi pour donner la différenciation entre deux sujets qui sont importants. Même s'il y a une hiérarchie qui montre dans l'exemple (5). On peut voir que la femme se traduit au pronom «itu » mais l'homme se traduit au pronom «sang ». On connaît la différence entre «itu » et « sang » maintenant, et il y a deux possibilités qui existent dans cet exemple. Le premier est pour donner la différenciation entre deux sujets qui sont important, et pour faire comprendre les lecteurs de différencier les deux sujets avec les pronoms différents. Le deuxième est pour souligner la hiérarchie qui existe entre la femme et l'homme a la référence d'histoire du roman.

De plus, l'article défini français dans le roman Syngué Sabour est aussi traduit au pronom "si". Le pronom "si" et "sang" sont dans le même type de pronom pour appeler quelqu'un ou quelque chose et donner la valeur plus forte. Le pronom "sang" a la valeur plus positive pour un sujet par contre, le pronom "si" est utilisé pour la même condition comme le pronom "sang" mais il donne la valeur plus négative. Par exemple :

(6) La prière faite, elle reste assise sur le tapis, pour écouter le mollah prêchant les hadith sur le jour de la semaine. (Rahimi, 2008, p. 23)

Selesai salat, ia tetap duduk di atas sajadah, mendengarkan si Mullah mengkhotbahkan hadis tentang hari dalam satu minggu. (Rahimi, 2012, p. 12)

(7) Le porteur d'eau frappe à la porte du voisin. (Rahimi, 2008, p. 26)

Si pengantar air mengetuk pintu rumah si tetangga. (Rahimi, 2012, p. 15)

En regardant les deux exemples, on peut voir la différence entre les deux est le caractère qui réfère le pronom "si". Le premier exemple montre un homme s'appelle Mollah qui se traduit en indonésien comme Si Mullah. Dans ce roman le Mollah est un caractère qui est détesté par la femme, le caractère principal. La femme déteste le Mollah parce qu'il la force toujours de prier sans arrêt pour son mari. Ce Mollah accuse la femme pour ne pas prier bien pour son mari, donc son mari ne se réveille pas encore. La présence du Mollah est toujours mal pour la femme. C'est la raison de la traduction "si" est équivalent pour l'article défini "le" du nom Mollah. Cette condition montre la valeur négative de Mollah, le caractère négatif pour la femme. Donc, la traduction du pronom "si" se choisit pour donner l'explication de condition négative pour le Mollah. Le deuxième exemple montre la hiérarchie entre la structure principale et le caractère secondaire. Le porteur et le voisin ne montrent que rarement dans ce roman, leur rôle n'est pas important mais ils sont existés. On peut dire, le choix du pronom "si" pour l'article défini "le" dans "le porteur" et "le voisin" pour marquer ou préciser le rôle de ce caractère dans le roman. Alors, la position entre le porteur et le voisin en comparant la femme et l'homme sont claires.

La présence de traduction zéro ou n'a pas d'équivalence dans ce roman est la plus haute. On divise la traduction qui n'a pas d'équivalence en indonésien dans les deux types : réel et suivi par l'explication. La traduction qui n'a pas d'équivalence réel est la traduction de l'article français en indonésien qui ne peut pas trouver le mot équivalent, mais ce nom est appartenu au nom qui n'est pas obligatoire d'avoir un article ou être interdit d'avoir un article. Puis, la traduction qui n'a pas d'équivalence suivie par l'explication est quand cet article n'est pas obligatoire parce que son existence se défini par l'explication et le point de vue du locuteur qui est compris par l'interlocuteur le nom qui est dit dans l'article. La traduction d'article zéro est une structure de la langue indonésienne, qui n'a besoin pas des catégories de l'article pour chaque nom obligatoire. 


\section{Conclusions}

La traduction de l'article défini français en indonésien dans le roman Syngué Sabour se divise dans les trois catégories. Ce sont la traduction zéro (qui n'a pas d'équivalence) et la traduction de pronom démonstratif «itu » et de pronom "sang" et "si". La traduction de pronom démonstratif «itu » défine le nom singulier qui est défini par l'explication en avant ou après.

«Sang» et «si » sont les pronoms qui précisent la valeur d'un caractère aux autres caractères. Ils peuvent montrer la gradation de caractère négative pour "si" et le caractère positif pour «sang ». Le caractère ou la valeur positive qui se dessin par "sang" ne montre pas toujours un caractère gentil, mais c'est plutôt une hiérarchie qui est plus haute parmi les autres caractères. Le pronom "si" marque la frontière entre les caractères principales et les caractères moins importants. La valeur négative comme le point de vue négatif d'un caractère est aussi possible être défini par le pronom "si".

La traduction zéro réel qui n'a pas d'équivalence dans la langue cible et la traduction zéro qui est suivie par la proposition + le nom qui explique que cet article appartient au nom qui le suit. La traduction «itu » signifie que le nom est défini et singulier. La traduction "sang et si" signifie la valeur du nom avec la condition et la relation entre les personnages. La traduction qui n'a pas d'équivalence (zéro) est un caractère typique de la langue indonésienne dont le nom n'est pas toujours obligatoire à avoir l'article pour marquer la référence et la situation d'un nom.

\section{Références}

Baskoro, S. (1992). Pemarkah Tanmaujud dalam Sistem Ketakrifan Bahasa Indonesia. [these] Universitas Gadjah Mada, Yogyakarta.

Givon, T. (1984). Syntax: A Functional Typological Introduction. Philadelphia: John Benjamins Publishing. Kamus Besar Bahasa Indonesia (KBBI). (2019). Sang. Retrieved from https://kbbi.web.id/sang

Kridalaksana, H. (1982). Kamus Linguistik. Jakarta: Gramedia Pustaka Utama.

Moeliono, A. M. (1988). Tata Bahasa Baku Bahasa Indonesia. Jakarta: Balai Pustaka.

Newmark, P. (1988). A Textbook of Translation. Text. New York: Prentice Hall.

Rahimi, A. (2008). Syngué Sabour. Pierre de patience. Paris: POL.

Rahimi, A. (2012). Batu Kesabaran Singge Sabur. (F. I. Mokoginta, Trans.). Yogyakarta : Jalasutra.

Sudaryanto. (1993). Metode dan Aneka Teknik Analisis Bahasa: Pengantar Penelitian Wahana

Kebudayaan Secara Linguistis. Yogyakarta : Duta Wacana University Press. 